\title{
RELAÇÃO ENTRE A ALIMENTAÇÃO COMPLEMENTAR E A PREVALÊNCIA DE EXCESSO DE PESO EM PRÉ-ESCOLARES
}

\section{RELATIONSHIP BETWEEN SUPPLEMENTARY FEEDING AND THE PREVALENCE OF OVERWEIGHT IN PRESCHOOLERS}

\author{
Franciélly Stadler', Priscila Antunes Tsupal'2, \\ Marcela Komechen Brecailo ${ }^{3}$, Daniele Gonçalves Vieira ${ }^{4}$
}

\section{RESUMO}

A prevalência da obesidade tem crescido e representa um problema de saúde pública. 0 objetivo dessa pesquisa foi avaliar a relação entre a alimentação complementar e a prevalência de sobrepeso/ obesidade em duas escolas de Imbituva-PR, sendo uma pública e outra particular. Caracterizase por um estudo transversal que avaliou o estado nutricional de 45 crianças de 4 a 6 anos de idade. A análise estatística foi realizada pelo Software SPSS 16.0. A média do IMC foi de 16,71 \pm $1,968\left(\mathrm{~kg} / \mathrm{m}^{2}\right)$. Apenas $15(33,3 \%)$ crianças foram amamentadas exclusivamente até o sexto mês de vida e esse não se mostrou um fator de proteção para sobrepeso/obesidade $(p=1,00)$. 0 tempo de aleitamento materno menor que um ano apresentou associação estatisticamente significativa com sobrepeso/obesidade $(p=0,027)$. A alimentação complementar não apresentou significância estatística quando comparada com sobrepeso/obesidade, entretanto foi precoce em quase todas as crianças pesquisadas.

Descritores: Nutrição da Criança; Consumo Alimentar; Escolar; Sobrepeso, Obesidade.

\section{ABSTRACT}

The prevalence of obesity has been increasing and it represents a public health issue. The aim of this research was to evaluate the relation between the complementary feeding and the overweight/ obesity prevalence in two schools from Imbituva-PR, a public one and a private one. It is characterized as a transversal study that evaluated the nutritional condition of forty-five children from 4 to 6 years old through anthropometric measures. The statistical analysis was performed using the SPSS 16.0 software. The average BMI was of $16.71 \pm 1,968\left(\mathrm{~kg} / \mathrm{m}^{2}\right)$. Only $15(33,3 \%)$ children were exclusively breastfed until the sixth month of life and it did not prove to be a protective element against obesity. The breastfeeding that has lasted less than one year showed a significant statistical association with overweight/obesity $(p=0,027)$. The complementary food did not present significant statistical results when compared to overweight/obesity. However, obesity was precocious in nearly all children studied.

Descriptors: Child Nutrition; Food Consumption; Child; Overweight; Obesity.
${ }^{1}$ Especialista em Nutrição Clínica pela Faculdade Evangélica do Paraná (FEPAR), Curitiba, PR, Brasil.

2 Doutora em Medicina Interna pela Universidade Federal do Paraná, (UFPR), Curitiba, PR, Brasil.
${ }^{3}$ Mestre em Nutrição pela Universidade Federal de Santa Catarina (UFSC), Florianópolis, SC, Brasil.

${ }^{4}$ Mestre em Pediatria e Ciências
Aplicadas à Pediatria pela
Universidade Federal de São Paulo
(UNIFESP), São Paulo, SP, Brasil.




\section{Introdução}

A prevalência do sobrepeso e da obesidade em crianças e adolescentes tem crescido de forma alarmante nos últimos anos e representa um problema de saúde pública relevante nos países desenvolvidos e em desenvolvimento, provocando um aumento de doenças associadas e a incapacidade na vida adulta, ${ }^{1,3}$.

Segundo a Organização Mundial da Saúde (OMS) em 2014, aproximadamente, 1,9 bilhões de adultos no mundo estão em condição de excesso de peso, destes mais de 600 milhões são obesos ${ }^{4}$.

O Índice de Massa Corporal (IMC) mede a razão entre o peso atual $(\mathrm{kg})$ e o quadrado da estatura $\left(\mathrm{m}^{2}\right)$ indicando diminuição do peso corporal, eutrofia ou aumento da massa corpórea. O aumento do IMC é um fator de risco para doenças não transmissíveis, como doenças cardiovasculares, diabetes, lesões musculoesqueléticas, sobrepeso, obesidade e alguns tipos de câncer ${ }^{4}$. Estudos atuais confirmam o aumento do IMC em crianças, indicando a importância das estratégias de promoção de estilos de vida mais saudáveis, enfatizando a adoção de uma alimentação mais equilibrada e a prática de atividade física regular ainda na infância e na adolescência ${ }^{1,3,5,6}$.

O aleitamento materno é uma das estratégias de promoção à saúde, prevenção de agravos e promoção da qualidade de vida. Evita o aparecimento do sobrepeso e da obesidade durante a infância, independentemente da idade da criança, da renda familiar, do estado nutricional e da escolaridade dos pais ${ }^{7}$. Promove bons hábitos alimentares que servirão para a profilaxia das doenças crônicas degenerativas na fase adulta ${ }^{8}$.

Até os seis meses de vida, o aleitamento materno exclusivo é capaz de alimentar, nutrir e proteger adequadamente a criança. A partir desse período, a introdução de novos alimentos é necessária para complementar a amamentação, bem como elevar a densidade energética da dieta e aumentar o aporte de micronutrientes ${ }^{9}$.

Devido àalta velocidade de crescimento, a criança pequena é vulnerável a erros, deficiências e excessosalimentares, muitas vezes irreversíveis. A introdução de novos alimentos envolve fatores biológicos, culturais e socioeconômicos, que determinam quando, como e porque iniciar a alimentação complementar ${ }^{10}$.

A correta introdução da alimentação complementar e a sua continuidade refletem o ganho de peso adequado, bem como o controle da obesidade na infância, adolescência e fase adulta ${ }^{11,12}$.

O objetivo dessa pesquisa foi avaliar a relação entre a alimentação complementar e a prevalência de sobrepeso/ obesidade em crianças pré-escolares, de duas escolas do município de Imbituva-PR, sendo uma pública e outra particular.

\section{Metodologia}

Caracteriza-se por um estudo transversal realizado com crianças pré-escolares em duas escolas do município de Imbituva, Estado do Paraná, sendo uma pública e outra privada. Amostragem foi por conveniência onde os elementos foram incluídos sem probabilidades previamente conhecidos permitindo a vantagem da escolha de amostra ${ }^{13}$.

Os critérios de inclusão foram: crianças com idade entre 4 e 6 anos, de ambos os gêneros, matriculadas no jardim II (Educação Infantil) e no primeiro ano do Ensino Fundamental, residentes na cidade de Imbituva - PR. Os critérios de exclusão foram alunos cujos pais se recusaram a assinar o Termo de Consentimento Livre e Esclarecido (TCLE).

Para verificação do estado antropométrico dos participantes, foram aferidos peso e a estatura segundo a metodologia proposta pelo Sistema de VigilânciaAlimentar e Nutricional (SISVAN) ${ }^{14}$ e calculado o Índice de Massa Corporal (IMC). A classificação e diagnóstico foram realizados por meio dos índices IMC para idade (IMC/I), peso para idade (P/I) e estatura para a idade (E/I), com o auxílio do Programa Anthro Plus versão $1.0 .4^{15} \mathrm{e}$ valores propostos pela WHO (2006).

Os instrumentos utilizados para coleta de dados foi o questionário de frequência alimentar que avaliou a alimentação atual da criança e o de avaliação da alimentação complementar, que avaliou o consumo alimentar nos primeiros dois anos de vida da criança. O segundo questionário avaliou também a duração do aleitamento materno e aleitamento materno exclusivo, idade da introdução de alimentos complementares, idade e escolaridade dos pais, informações sobre o cuidador da criança, trabalho materno, idade que a criança começou a frequentar a creche e os conhecimentos da família sobre alimentação saudável. Os pais e responsáveis tiveram participação direta no preenchimento dos questionários após o conhecimento e assinatura do TCLE.

Foi realizado um estudo para avaliar a aplicabilidade do instrumento em relação aos objetivos do estudo, onde participaram 15 crianças, as quais não entraram na amostra final. 
Os dados foram digitados em dupla entrada no Software Excel® e exportados posteriormente para o Pacote Estatístico Epi Info® versão 3.5.2. Com auxílio da ferramenta Data Compare realizou-se a averiguação e a correção das planilhas digitadas. A análise estatística descritiva foi realizada por meio de porcentagens, frequências, médias e desvio-padrão. $O$ teste Qui Quadrado foi realizado com variáveis qualitativas através do Pacote Estatístico SPSS 16.0.

A pesquisa foi aprovada pelo Comitê de Ética em Pesquisa da UNICENTRO, anteriormente a data a coleta de dados, respeitando assim as questões éticas envolvidas e descritas na Resolução CNS 196/96, protocolo número (006/2012).

\section{Resultados e Discussão}

Foram encaminhados aos pais 110 questionários, sendo que 60 foram preenchidos e assinados. Destes, 6 apresentaram dados inconsistentes e 9 crianças faltaram ao dia da avaliação antropométrica, perfazendo um total de 45 crianças participantes. Dessas, 46,7\% ( $n=21)$ eram meninas e 53,3\% $(n=24)$ meninos, $42,2 \%(n=19)$ estavam matriculados na escola privada e $57,8 \%(n=26)$ na escola pública. A média de idade das crianças foi de $69( \pm 5,48)$ meses.

Com relação ao perfil dos pais que responderam ao questionário, entre as mães, $60 \%(n=27)$ tinham entre 20 a 34 anos, 35,6\% ( $n=16)$ tinham 35 anos ou mais e 4,4\% ( $n=2)$ não responderam. Entre os pais, $53,3 \%(n=24)$ tinham entre 20 e 34 anos, $44,4 \%(n=20)$ tinham 35 anos ou mais e 2,2\% ( $n=1)$ não respondeu.

Analisando a escolaridades dos pais, conforme os dados da figura 1, a maioria das mães tinha ensino médio completo $(22,2 \% ; n=10)$ e a maioria dos pais tinha o ensino fundamental incompleto $(33,3 \% ; n=15)$.

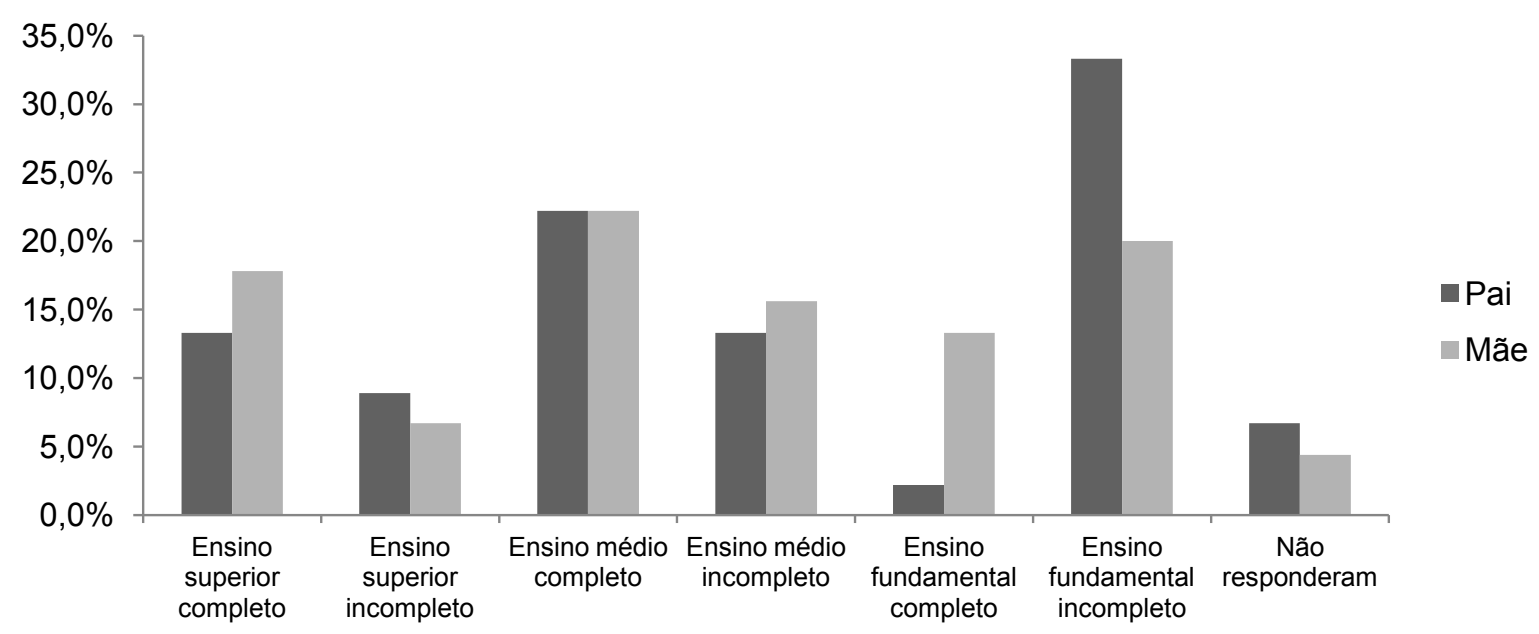

Figura 1. Escolaridade paterna e materna de crianças entre 4 e 6 anos de idade, ambos os gêneros, matriculadas no jardim II (Educação Infantil) e no primeiro ano do Ensino Fundamental. Imbituva - PR.

Com relação à renda, $51,1 \%(n=23)$ recebiam em torno de 1 a 3 salários mínimos por mês, $15,6 \%(n=7)$ de 0,5 a 1 salário mínimo, $13,3 \%(n=6)$ mais que 6 salários mínimos, $11,1 \%(n=5)$ de 3 a 5 salários mínimos, e 8,9\% ( $n=4)$ optaram por não responder a pergunta. Um estudo realizado por Barbosa e Colaboradores ${ }^{10}$ verificou que a renda familiar inferior a três salários mínimos é um dos fatores de risco independentes para o desmame precoce. Destaca-se na literatura a relação entre a maior escolaridade das mães e a maior renda familiar ao sobrepeso e a obesidade ${ }^{16}$.

Entre as 45 mães, $51,1 \%(n=23)$ trabalhavam fora de casa, 44,4\% (n=20) não trabalhavam fora e 4,4\% (n=2) não responderam. Quanto ao cuidador da criança, $40 \%(n=18)$ relataram ser os pais da criança, $12(26,7 \%)$ a mãe, $7(15,6 \%)$ contavam com a ajuda de uma babá, 15,6\% $(n=7)$ recebiam cuidados de avós ou tios e 2,2\% $(n=1)$ não respondeu. Das crianças pesquisadas, 6,7\% ( $n=3$ ) foram matriculadas no Centro de Educação Infantil com 1 ano, 2,2\% ( $n=1)$ com 2 anos, 20,0\% ( $n=9)$ com 3 anos, 28,9\% ( $n=13)$ com 4 anos, 33,3\% $(n=15)$ com 5 anos e 6,7\% $(n=3)$ com 6 anos.

Quando questionados sobre o interesse de buscar informações sobre alimentação saudável, $33,3 \%(n=15)$ das famílias pesquisadas relataram não buscar informação em nenhuma fonte de dados. Entre as 30 (67\%) famílias que 
buscavam informações, a televisão e a internet tiveram a maior influencia com $56,7 \%$ ( $n=17)$ e $23,3 \%(n=7)$ respectivamente, seguida pelas revistas $10 \%(n=3)$, pela escola $6,7 \%(n=2)$ e profissional nutricionista 3,3\% $(n=1)$. Estudos mostram que a falta de conhecimento em nutrição é fator de risco para a obesidade e a introdução precoce de alimentos ${ }^{10}$. Ressalta-se o grande número de pais que buscam informações na internet, televisão e revistas acarretando um possível risco para família por se tratar de informações não cientificas e de fontes não seguras.

Do total de crianças avaliadas, $60 \%(n=27)$ eram eutróficas e $40 \%(n=18)$ estavam com excesso de peso. Por uma questão metodológica do estudo, os valores de sobrepeso e obesidade foram agrupados devido ao pequeno número da amostra. A média do peso (em $\mathrm{kg}$ ) foi de $22,05 \pm 3,96$ e a média da estatura (em metros) de 1,14 $\pm 0,05$. A média do percentil do IMC foi de 71,12 ( $\pm 24,27$ ), estando acima do adequado (percentil 50). Não houve diferença estatística significativa no perfil antropométrico entre as crianças da escola pública e privada ( $p=0,324)$, como mostra a tabela 1. Por outro lado Paula e colaboradores $(2014)^{17}$ encontraram uma alta prevalência de excesso de peso tanto em escolas públicas como particulares, com maiores valores para crianças da rede particular

Tabela 1 - Estado nutricional segundo o IMC para idade e fatores associados, Imbituva, 2012

\begin{tabular}{|c|c|c|c|}
\hline & $\begin{array}{l}\text { eutrofia } \\
\text { n (\%) }\end{array}$ & $\begin{array}{c}\text { sobrepeso/obesidade } \\
\text { n (\%) }\end{array}$ & $\mathbf{P}$ \\
\hline \multicolumn{4}{|l|}{ Escola } \\
\hline Escola pública & $14(53,8 \%)$ & $12(46,2 \%)$ & \multirow{2}{*}{$0,324^{*}$} \\
\hline Escola particular & $13(68,4 \%)$ & $6(31,6 \%)$ & \\
\hline \multicolumn{4}{|c|}{ Aleitamento materno exclusivo } \\
\hline 6 meses & $9(60,0 \%)$ & $6(40,0 \%)$ & \multirow[t]{2}{*}{$1,000^{*}$} \\
\hline$<6$ meses & $18(60,0 \%)$ & $12(40,0 \%)$ & \\
\hline \multicolumn{4}{|c|}{ Tempo de aleitamento materno } \\
\hline 1 ano ou mais & $12(46,2 \%)$ & $14(53,8 \%)$ & \multirow[t]{2}{*}{$0,027^{* * *}$} \\
\hline$<1$ ano & $15(78,9 \%)$ & $4(21,1 \%)$ & \\
\hline
\end{tabular}

* teste Qui-quadrado; ** diferença estatisticamente significativa.

Entre as 45 crianças avaliadas, apenas $33,3 \%(n=15)$ foram amamentadas exclusivamente até o sexto mês de vida. O tempo de aleitamento materno exclusivo foi em média 3,7 meses, inferior ao recomendado pelo Ministério da Saúde ${ }^{18}$. e pela Organização Mundial de Saúde ${ }^{19}$, que preconiza que 0 aleitamento materno seja exclusivo por seis meses. Esses resultados foram semelhantes a outros estudos encontrados na literatura ${ }^{20,21,22,7}$.

De acordo com a Tabela 1, 0 aleitamento materno exclusivo até o sexto mês de vida não foi um fator de proteção contra sobrepeso/obesidade $(p=1,000)$, como observado também em alguns estudos encontrados na literatura ${ }^{25,26,27}$. Em contrapartida, outros estudos evidenciaram que o aleitamento materno exclusivo por 6 meses é um fator protetor contra a obesidade ${ }^{7,22,23,24}$. O resultado do presente estudo pode estar associado ao tamanho pequeno da amostra. Ressalta-se a importância da continuidade de estudos que evidenciem essa questão.

Uma revisão sistemática recente, evidencia algumas vantagens do aleitamento materno exclusivo por 6 meses, como menor risco de infecção gastrointestinal, perda de peso materno mais significativa após o nascimento, e retorno tardio de períodos menstruais. Semelhantemente ao presente estudo, o aleitamento materno exclusivo até seis meses não se mostrou como protetor contra a obesidade ${ }^{28}$.

A prevalência do aleitamento materno em crianças de zero a seis meses foi $15,6 \%$ ( $n=7)$, de 6 a 12 meses foi de 22,2\% ( $n=10)$, de 1 a 2 anos de $40 \%$ ( $n=18)$ e 17,8\% $(n=8)$ acima de 2 anos. Apenas duas crianças (4,4\%) não foram amamentadas no peito.

0 aleitamento materno por um ano ou mais apresentou efeito protetor contra o sobrepeso/obesidade $(p=0,027)$ de acordo com os dados da Tabela 1. Estudos da literatura corroboram com esses dados ${ }^{7,29}$. Frente ao aumento da prevalência da obesidade e ao seu difícil tratamento, estratégias simples e de baixo custo que permitam a sua prevenção devem ser 
enfatizadas. A hipótese de que o aleitamento materno teria um efeito protetor contra a obesidade não é recente. Porém, resultados controversos têm sido encontrados, fazendo com que o tema permaneça atual. Os inúmeros benefícios do aleitamento materno descritos na literatura justificam a sua recomendação em períodos preconizados pela WHO ${ }^{29,30,31 . ~}$

A introdução alimentar precoce pode contribuir para o risco de excesso de peso ${ }^{23}$. Na amostra estudada houve uma introdução precoce, antes dos seis meses de vida da criança, de quase todos os alimentos pesquisados. 0 chá foi o alimento introduzido mais precocemente, em média aos 4,4 2 2,38 meses em 51,1\% ( $n=23)$ crianças, seguido da água $48,9 \%(n=22)$, da papa doce $24,5 \%(n=11)$, do leite de vaca $22,2 \%(n=10)$, do iogurte $22,2 \%(n=10)$, sopa de legumes $17,8 \%(n=8)$, cereais e tubérculos $11,1 \%(n=5)$, hortaliças $11,1 \%(n=5)$, açúcar $8,9 \%(n=4)$, mel $8,9 \%(n=4)$, frango ou peixe $6,7 \%(n=3)$, comida da família 4,4\% ( $n=2)$, carnes bovina, e ovos 2,2\% ( $n=1)$. Resultados semelhantes têm sido encontrados em outras pesquisas ${ }^{7,10,20,32}$.

Tabela 2 - Idade da introdução de alguns alimentos na população estudada, Imbituva, 2012

\begin{tabular}{c|c|c|c|c|c}
\hline Alimento & $\begin{array}{c}\text { Menor de seis } \\
\text { meses }\end{array}$ & $\begin{array}{c}\text { Seis a oito } \\
\text { meses }\end{array}$ & $\begin{array}{c}\text { Nove meses } \\
\text { a } 1 \text { ano }\end{array}$ & $\begin{array}{l}\text { Acima de 1 } \\
\text { ano }\end{array}$ & $\begin{array}{c}\text { Não Ingeriu/ } \\
\text { Não Respondeu }\end{array}$ \\
\hline Achocolatado & $0(0 \%)$ & $10(22,2 \%)$ & $9(20 \%)$ & $16(35,6 \%)$ & $10(22,2 \%)$ \\
\hline Açúcar & $4(8,9 \%)$ & $16(35,6 \%)$ & $10(22,2 \%)$ & $8(17,7 \%)$ & $7(15,6 \%)$ \\
\hline Água & $22(48,9 \%)$ & $22(48,9 \%)$ & $0(0 \%)$ & $0(0 \%)$ & $1(2,2 \%)$ \\
\hline Cereais & $5(11,1 \%)$ & $26(57,7 \%)$ & $9(20 \%)$ & $4(8,9 \%)$ & $1(2,2 \%)$ \\
\hline Bolacha & $5(11,1 \%)$ & $32(71,1 \%)$ & $6(13,3 \%)$ & $1(2,2 \%)$ & $1(2,2 \%)$ \\
\hline Carnes & $3(6,7 \%)$ & $21(46,7 \%)$ & $11(24,4 \%)$ & $7(15,5 \%)$ & $3(6,7 \%)$ \\
\hline Chá & $23(51,1 \%)$ & $15(33,3 \%)$ & $0(0 \%)$ & $0(0 \%)$ & $7(15,6 \%)$ \\
\hline Comida da família & $2(4,4 \%)$ & $8(26,3 \%)$ & $20(44,4 \%)$ & $10(22,2 \%)$ & $1(2,2 \%)$ \\
\hline Embutidos & $0(0 \%)$ & $4(8,8 \%)$ & $15(33,3 \%)$ & $21(46,7 \%)$ & $5(11,1 \%)$ \\
\hline Guloseimas & $0(0 \%)$ & $12(26,7 \%)$ & $18(40 \%)$ & $10(22,2 \%)$ & $5(11,1 \%)$ \\
\hline Hortaliças & $5(11,1 \%)$ & $27(60 \%)$ & $4(8,9 \%)$ & $5(11,1 \%)$ & $4(8,9 \%)$ \\
\hline logurte & $10(22,2 \%)$ & $27(60 \%)$ & $7(15,6 \%)$ & $0(0 \%)$ & $1(2,2 \%)$ \\
\hline Leite de vaca & $10(22,2 \%)$ & $16(35,6 \%)$ & $10(22,2 \%)$ & $6(13,3 \%)$ & $3(6,6 \%)$ \\
\hline Mel & $4(8,9 \%)$ & $3(6,7 \%)$ & $10(22,2 \%)$ & $8(17,8 \%)$ & $20(44,4 \%)$ \\
\hline Ovo & $1(2,2 \%)$ & $15(35,6 \%)$ & $9(20,0 \%)$ & $13(28,9 \%)$ & $6(13,3 \%)$ \\
\hline Papa doce & $11(24,5 \%)$ & $24(53,3 \%)$ & $5(11,1 \%)$ & $0(0 \%)$ & $5(11,1 \%)$ \\
\hline Sopa de legumes & $8(17,8 \%)$ & $29(64,7 \%)$ & $2(4,4 \%)$ & $2(4,4 \%)$ & $4(8,9 \%)$ \\
\hline
\end{tabular}

A adequada alimentação complementar tem um efeito imediato na saúde infantil e se estende até os dois anos de idade da criança participando ativamente do seu crescimento e desenvolvimento. A introdução precoce de alimentos aumenta a vulnerabilidade da criança a infecções, diarreias e desnutrição e a introdução tardia aumenta o risco das deficiências nutricionais ${ }^{32}$.

Analisando a frequência da alimentação atual das crianças estudadas, os alimentos ricos em Vitamina A mais consumidos diariamente foram o leite integral $53,3 \%(n=24)$, o ovo $11,1 \%(n=5)$, a cenoura $8,9 \%(n=4)$ e a couve $6,7 \%$ $(n=3)$. A Vitamina A é essencial ao crescimento e desenvolvimento do ser humano, atuando na manutenção da visão, no funcionamento adequado do sistema imunológico e nas mucosas. O Ministério da Saúde preconiza que o consumo de vitamina A seja de 500ug, para crianças de 4 a 6 anos $^{33}$.

Entre os alimentos ricos em ferro os mais consumidos diariamente pela população estudada foram o feijão $57,8 \%$ ( $n=26)$, a carne bovina 15,6\% ( $n=7)$ e a carne de frango 13,3\% ( $n=6)$. O ferro é um nutriente de extrema importância para a vida, atuando na síntese das células vermelhas do sangue e no transporte do oxigênio para todas as células do corpo. A anemia em crianças pode estar associada a pouco tempo de aleitamento materno exclusivo, alimentação prolongada 
com leite de vaca e com a introdução da alimentação complementar precoce ${ }^{33}$. Evidencia-se no presente estudo a possibilidade de risco de carência deste nutriente por várias famílias. Outros autores evidenciam que a introdução precoce do leite de vaca aumenta o risco de a criança apresentar deficiência de ferro ${ }^{34}$.

Quanto aos alimentos ricos em vitamina C, $24,4 \%(n=11)$ crianças consumiam diariamente 0 suco de laranja, $13,3 \%(n=6)$ a laranja (fruta) e 4,4\% (n=2) o suco de limão. O consumo de alimentos fonte de vitamina $C$ potencializa a absorção do ferro proveniente de produtos vegetais (folhosos verdes escuros, brócolis, entre outros) auxiliando também no fortalecimento da imunidade do corpo ${ }^{33}$.

Observou-se um consumo diário considerável de sódio, principalmente em produtos industrializados, sendo que 44,4\% ( $n=20)$ das crianças estudadas consumiram suco artificial, $15(33,3 \%)$ achocolatado, 24,4\% ( $n=11)$ macarrão instantâneo, 20,0\% ( $n=9)$ salgadinho tipo chips ${ }^{\circledR}, 15,6 \%(n=7)$ refrigerante e 15,6\% ( $\left.n=7\right)$ mortadela. O sódio está presente naturalmente nos alimentos, porém a maior parte é adicionada por consumidores, produtores e manipuladores, durante 0 consumo, fabricação e preparo dos alimentos, na forma de sal, para melhorar o sabor ou para preservação dos alimentos. O seu consumo excessivo está associado ao desenvolvimento da hipertensão arterial, doenças cardiovasculares, renais entre outras. A recomendação atual para a população brasileira em geral é de $2000 \mathrm{mg}$ de sódio por dia, o que equivale a $5 \mathrm{~g}$ de sal ${ }^{33}$. Um estudo realizado por Matuk e Colaboradores ${ }^{35}$ corrobora com 0 atual quando os autores, avaliando a composição das lancheiras dos alunos em uma escola particular, observaram um alto consumo de bebidas e alimentos industrializados, ricos açúcares, gorduras e sódio.

Entre os alimentos ricos em fibras, o feijão foi consumido diariamente por $26(57,8 \%)$ crianças, seguido pela banana $16(35,6 \%)$, tomate $12(26,7 \%)$, alface $10(22,2 \%)$, maçã $8(17,8 \%)$ e arroz integral $5(11,1 \%)$. Estudos mostram que o baixo consumo de fibras associa-se ao sobrepeso e obesidade ${ }^{36}$.

Algumas limitações foram encontradas no presente estudo como a dificuldade de aferir outras variáveis que poderiam interferir no peso atual da criança, tais como peso ao nascer, ingestão energética atual e o nível de atividade física da criança e o esquecimento por parte dos pais no preenchimento do questionário sugerindo um possível viés de análise. Outra questão é a limitação em relação ao tamanho pequeno da amostra para justificar ou não a proteção do aleitamento materno exclusivo até os seis meses, por outro lado, mesmo com a amostra reduzida, o estudo mostrou que 0 aleitamento materno por mais de um ano foi fator protetor contra o sobrepeso/obesidade.

\section{Considerações Finais}

O aleitamento materno exclusivo não foi um fator de proteção contra a obesidade. Em contrapartida, o aleitamento materno por um ano ou mais se mostrou como fator de proteção contra o sobrepeso/obesidade entre as crianças estudadas. Os alimentos complementares foram introduzidos precocemente em quase todos os casos estudados. Ressalta-se, portanto, a importância da continuidade e do fortalecimento das ações de promoção, proteção e apoio ao aleitamento materno e das práticas alimentares corretas como precursores de hábitos de vida saudáveis na infância, adolescência e na vida adulta.

\section{Referências}

1. Onis M. Prevenção do sobrepeso e da obesidade infantis. J Pediat. 2015;91(2):105-107.

2. Chaves APB, Queiroz LFR, Abreu MA, Medeiros KB. Sobrepeso e obesidade infantil - um problema de saúde pública em escolares de norte a sul do país Enferm. Bras. 2011;10(6):371-6.

3. Souza MCC, Tibúrcio JD, Bicalho JMF, Rennó HMS, Dutra JS, Campos LG et al. Fatores associados à obesidade e sobrepeso em escolares. Texto Contexto Enferm. 2014;23(3):712-9.

4. World Health Organization. Obesity and overweight. Geneva: WHO; 2015.

5. Cabrera TFC, Correia IFL, Santos DO, Pacagnelli FL, Prado MTA, Silva TD et al. Análise da prevalência de sobrepeso e obesidade e do nível de atividade física em crianças e adolescentes de uma cidade do sudoeste de São Paulo. Journal of Human Growth and Development. 2014;24(1): 67-66.

6. Reis CEG, Vasconcelos IAL, Barros JFN. Políticas públicas de nutrição para o controle da obesidade infantil. Rev Paul Pediatr. 2011;29(4):625-33. 
7. Simon VGN, Souza JMP, Souza SB. Aleitamento materno, alimentação complementar, sobrepeso e obesidade em pré-escolares. Rev Saude Publica. 2009;43(1):60-69.

8. Pereira PF , Alfenas RCG, Araújo RMA. O aleitamento materno influencia o risco de desenvolvimento de diabetes mellitus na criança? Uma análise das evidências atuais. J Pediatr. 2014;90(1):7-15

9. Mais LA, Domene SMA, Barbosa MB, Taddei JAAC. Diagnóstico das práticas de alimentação complementar para 0 matriciamento das ações na Atenção Básica. Ciência \& Saúde Coletiva. 2014;19(1):93-104.

10. Barbosa MB, Palma D, Domene SMA, Taddei JAAC. Lopez FA. Fatores de risco associados ao desmame precoce e ao período de desmame em lactentes matriculados em creches. Rev. paul. pediatr. 2009;27(3):272-281.

11. Caetano MC, Ortiz TTO, Silva SGL, Souza FIS, Sarni OS. Alimentação complementar: práticas inadequadas em lactentes. J Pediatr. 2010;86(3):196-201.

12. World Health Organization. Infant and young child feeding: Model Chapter for textbooks for medical students and allied health professionals. Geneva: WHO; 2009.

13. Anderson DR, Sweeney DJ, Williams TA. Estatística aplicada à administração e economia. São Paulo: Editora Cengage Learning, 2007.

14. Brasil. Vigilância Alimentar e Nutricional. Orientações básicas para a coleta, processamento, análise de dados e informação em serviços de saúde. Brasília (DF): Ministério da Saúde; 2004.

15. World Health Organization. AnthoPlus v 1.0.4. Geneva: WHO; 2007.

16. Souza MCC, Tibúrcio JD, Bicalho JMF, Rennó HMS, Dutra JS, Campos LG. Fatores associados à obesidade e sobrepeso em escolares. Texto Contexto Enferm. 2014; 23(3):712-9.

17. Paula FAR, Lamboglia CMGF, Silva VTBL, Monteiro MS, Moreira AP, Pinheiro MHNP, Silva CAB. Prevalência de sobrepeso e obesidade em escolares da rede pública e particular da cidade de Fortaleza. Rev Bras Promoç Saúde. 2014;27(4):455-461.

18. Brasil. Ministério da Saúde. Saúde da criança: Nutrição infantil - Aleitamento materno e alimentação complementar. Brasília (DF): Ministério da Saúde; 2009.

19. World Health Organization. Collaborative Nutrient adequacy of exclusive breastfeeding for the term infant during the fi rts six months of life. Geneva: WHO; 2002.

20. Caetano MC, Ortiz TTO, Silva SGL, Souza FIS, Sarni ROS. Alimentação complementar: práticas inadequadas em lactentes. J Pediatr. 2010;86(3):196-201.

21. Corrêa EN, Corso ACT, Moreira EAM, Kazapi IMA. Alimentação complementar e características maternas de crianças menores de dois anos de idade em Florianópolis (SC). Rev Paul Pediatr. 2009;27(3):258-64.

22. Moraes JFVN, Giugliano R. Aleitamento materno exclusivo e adiposidade. Rev Paul Pediatr. 2011;29(2):152-6.

23. Castro MBT, Gigante DS, Silva LO, Nascimento BC, Padilha PC. Introdução de alimentos e excesso de peso em pré-escolares de uma comunidade vulnerável da cidade do Rio de Janeiro - Associação da introdução de alimentos e excesso de peso. Demetra. 2014; 9(3); 645-660.

24. Crume TL, Ogden LG, Mayer-Davis EJ, Hamman RF, Norris JM, Bischoff KJ et al. The impact of neonatal breastfeeding on growth trajectories of youth exposed and unexposed to diabetes in utero: the EPOCH Study. Int J Obes. 2012; 36(4):529-34.26.

25. Huus K, Ludvigsson JF, Enskär K, Ludvigsson J. Exclusive breastfeeding of Swedish children and its possible influence on the development of obesity: a prospective cohort study. BMC Pediatr. 2008;8(1):42.

26. Shields L, Mamun AA, O'Callaghan M, Williams GM, Najman JM. Breastfeeding and obesity at 21 years: a cohort study. J Clin Nurs. 2010;19(1):1612-1617.

27. Novaes JF, Lamounier JA, Colosimo EA, Franceschini SC, Priore SE. Breastfeeding and obesity in Brazilian children. Eur J Public Health. 2012;22(2):383-89.

28. Kramer MS, Kakuma R. Optimal duration of exclusive breastfeeding. Cochrane Database of Systematic Reviews 2012, Issue 8. Art. No.: CD003517. DOI: 10.1002/14651858.CD003517.pub2.

29. Marseglia L, Manti S, D’Angelo G, Cuppari C, Salpietro V, Filippelli M. et al. Obesity and breastfeeding: The strength of association. Women and Birth. 2015;28(2):81-86.

30. Daniels SR. Breastfeeding and risk of obesity. The Journal of Pediatrics. 2014; 164(6):1245-1247.

31. Péneau S, Hercberg S, Rolland-Cachera M. Breastfeeding, Early Nutrition, and Adult Body Fat. The Journal of Pediatrics. 2014; 164(6):1363-1368. 
32. Silva LMP, Venâncio SI, Marchioni DML. Práticas de alimentação complementar no primeiro ano de vida e fatores associados. Rev Nutr. 2010;23(6):983-992.

33. Brasil. Coordenação Geral de Alimentação e Nutrição. [acesso 2012 Jul 10] Disponível em: http://nutricao.saude.gov.br/. 34. Bortolini GA, Vitolo MR. Importância das práticas alimentares no primeiro ano de vida na prevenção da deficiência de ferro. Rev Nutr. 2010;23(6):1051-62.

35. Matuk TT, Stancari PCS, Bueno MB, Zaccarelli EM. Composição de lancheiras de alunos de escolas particulares de São Paulo. Rev Paul Pediatr. 2011;29(2):157-63.

36. Bernaud FSR, Rodrigues TC. Fibra alimentar - Ingestão adequada e efeitos sobre a saúde do metabolismo. Arq Bras Endocrinol Metab. 2013;57(6):397-405.

\section{Franciélly Stadler}

Endereço para correspondência - Rua: Joaquim Marcondes Pupo, $n^{\circ} 1056$,

Bairro: Centro, CEP: 84430-000, Imbituva, PR, Brasil.

E-mail: franstadler@hotmail.com

Lattes: http://lattes.cnpq.br/5189203118589820

Priscila Antunes Tsupal - ptsupal@gmail.com

Marcela Komechen Brecailo - marbrecailo@gmail.com

Daniele Gonçalves Vieira - daniele.gonvieira@gmail.com

Enviado em 23 de outubro de 2014. Aceito em 13 de dezembro de 2015. 